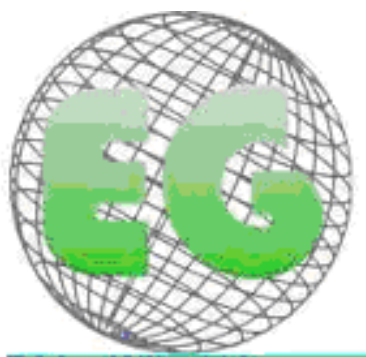

$N^{\circ} 18$
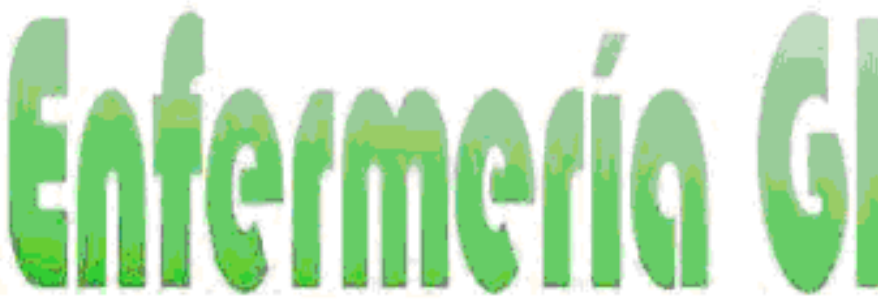

Revista electrônica cuatrimestral de Enfermeria

Febrero 2010

www.um.es/egloball

\title{
CLÍNICA
}

\section{DIAGNÓSTICOS DE ENFERMERÍA DE COMPLICACIONES EN LA SALA DE RECUPERACIÓN ANESTÉSICA}

DIAGNÓSTICOS DE ENFERMAGEM NAS COMPLICACÕ̃ES EM SALA DE RECUPERAÇÃO ANESTÉSICA

\begin{abstract}
*De Mattia, AL, **Faria Maia, L., "*Santos Silva, S., **De Oliveira, TC.
*Doutora em Enfermagem. Professora Adjunta. Universidade Minas Gerais.***aduada em Enfermagem. Universidade Católica de Santos. Brasil.
\end{abstract}

\author{
Palabras clave: Diagnóstico de Enfermería; Sala de Recuperación Anestésica \\ Palavras-chave: Diagnóstico de Enfermagem; Sala de Recuperação Pós-Anestésica \\ Keywords: Nursing Diagnoses; Post Anesthesia Care Unit.
}

\section{RESUMEN}

Este es un estudio descriptivo, exploratorio, con método cualitativo y rasgo no empírico, con análisis de frecuencia absoluta y relativa de los datos. Presenta como objetivo identificar los diagnósticos de enfermería según la Taxonomía II de la NANDA, en la Sala de Recuperación Post-Anestésica (SRPA). La muestra estaba constituida por 30 enfermos adultos, sometidos a cirugía selectiva, con anestesia general y evaluación de las condiciones físicas Americam Society of Anesthesiologists (ASA1). El campo de estudio fue la SPRA, la cual cuenta con 8 camas de un hospital de la red mixta de la ciudad de Santos. Los datos fueron colectados a través de un instrumento estructurado, con datos referentes al sexo, edad, diagnóstico médico, intervención anestésico quirúrgica, tiempo quirúrgico, tiempo anestésico, signos vitales, incluyendo verificación de la tensión arterial en la internación, índices de Aldrete Kroulik y complicaciones como náusea, vómito y dolor. Tras el análisis de los problemas de enfermería, fueron identificados los diagnósticos de enfermería, siendo los de mayor frecuencia la hipotermia, náusea y dolor agudo.

\section{RESUMO}

Este é um estudo descritivo, exploratório, com método quantitativo e delineamento não experimental, com análise de freqüência absoluta e relativa dos dados. Apresenta como objetivo identificar os diagnósticos de enfermagem, segundo a Taxonomia II da NANDA, na Sala de Recuperação PósAnestésica (SRPA). A amostra foi constituída de 30 pacientes adultos, submetidos à cirurgia eletiva, com anestesia geral, e avaliação das condições físicas Americam Society of Anesthesiologists (ASA 1). O campo de estudo foi a SRPA, a qual conta com 8 leitos, de um Hospital da rede mista da cidade de Santos. Os dados foram coletados por meio de um instrumento estruturado, com dados 
referentes ao sexo, idade, diagnóstico médico, intervenção anestésico-cirúrgica, tempo cirúrgico, tempo anestésico, sinais vitais incluindo verificação de pressão arterial na internação, avaliação do índice de Aldrete KrouliK; e complicações como náusea, vômito e dor. Após o levantamento dos problemas de enfermagem, foram identificados os diagnósticos de enfermagem, sendo os maior freqüência a hipotermia, náusea e dor aguda.

\section{ABSTRACT}

This is a descriptive, exploratory study with a qualitative method and non-experimental design, with analysis of absolute and relative frequency of data. It shows how to identify nursing diagnoses according to NANDA Taxonomy II in Post Anesthesia Care Unit (PACU). The sample consisted of 30 adult patients undergoing elective surgery with general anesthesia, and evaluation of physical conditions according to American Society of Anesthesiologists (ASA 1). The field of study was the PACU, which has 8 beds in a mixed network hospital in the city of Santos. Data were collected through a structured method, with data on gender, age, medical diagnoses, intervention-surgical anesthetic, surgical time, anesthetic time, vital signs including blood pressure at admission, assessment of the index of Aldrete KrouliK; and complications such as nausea, vomiting and pain. After the analysis of the nursing problems, nursing diagnoses were identified, with the greatest frequency being hypothermia, nausea, and acute pain.

\section{INTRODUCCIÓN}

El tema propuesto para esta investigación trata de los diagnósticos de enfermería (DE) de pacientes bajo efecto de anestesia general en Sala de Recuperación Post-Anestésica (SRPA) y sus complicaciones. La SRPA es el lugar destinado a la atención de pacientes en el post-operatorio inmediato hasta que se hayan recuperado y se encuentren en condiciones de regresar a su lugar de origen. ${ }^{(1)}$

Los pacientes en el postoperatorio inmediato presentan algunas complicaciones tales como respiratorias (hipoxia, hipercapnia, disnea); cardiovasculares (desvíos de tensión arterial, bradicardia o taquicardia); renales; neurológicas (alteraciones en el nivel de conciencia); neuromusculares, hidroelectrolíticas, y otras como hipotermia, dolor, náuseas y vómitos.

Los DE sirven de base para el juicio clínico ante las respuestas del paciente, de la familia y de la comunidad, pues a partir de tal juicio el profesional enfermero puede definir su plan de cuidados. Se trata de identificar las necesidades básicas del ser humano que precisan atención y de determinar el grado de dependencia de esta atención por tipo y extensión. En la propia conceptuación de enfermería se percibe que el diagnóstico incluye dos dimensiones: identificar las necesidades y determinar el grado de dependencia. ${ }^{(2)}$

En la 9a Conferencia de The North American Nursing Diagnosis Association (NANDA), realizada en 1990, fue aprobada la definición de los DE como: "Un juicio clínico sobre las respuestas del individuo, de la familia o de la comunidade a problemas de salud / procesos vitales reales o potenciales. El diagnóstico de enfermería sirve de base para seleccionar la intervención de enfermería que obtenga los resultados de los cuales el enfermero es responsable."(3)

EI DE se realiza a través de relatos e indicios del paciente y el profesional enfermero deberá tener conocimiento suficiente para clasificarlos y relacionarlos con los diagnósticos para poder definir el plan de cuidados que promueva la recuperación del paciente. Durante la recuperación anestésica, que incluye desde el momento del alta del paciente de la sala de operaciones hasta su alta de la recuperación anestésica, el paciente presenta varias 
alteraciones, ocasionadas tanto por el procedimiento quirúrgico como por el procedimiento anestésico, que deberán ser prevenidas, detectadas y tratadas.

Para Prado et al ${ }^{(4)}$ la SRPA es el lugar destinado a la atención intensiva del paciente, desde su salida de la sala de operaciones hasta la recuperación de la conciencia, eliminación de anestésicos y estabilización de señales vitales.

Possari ${ }^{(1)}$ sostiene que la SRPA tiene varias finalidades, entre ellas, ofrecer mejores condiciones de asistencia médica y de enfermería en el período postoperatario y postanestésico inmediato; reducir la mortalidad postanestésica y postoperatoria; facilitar las tareas de rutina en las unidades de ingreso hospitalario, que se eximen con la presencia de un recién operado en condiciones precarias; proporcionar más seguridad a los pacientes y familiares y disminuir los posibles accidentes postoperatorios y postanestésicos inmediatos. El enfermero a cargo de la sala de recuperación debe sistematizar el registro de las informaciones, manteniendo un vínculo activo con los profesionales de salud, además de ofrecer al equipo de enfermería condiciones para actuar con el cliente de forma efectiva, planificada y segura.

Con la utilización de una herramienta es más fácil completar los datos del paciente, se agilizan los registros y las condiciones físicas del paciente pueden valorarse de forma racionalizada. En consecuencia, la asistencia de enfermería en la Recuperación Postanestésica (RPA) se acerca al paciente y se vuelve más humanizada. ${ }^{(5)}$

Una de las formas de evaluar al paciente es clasificando sus condiciones físicas con un método desarrollado por The American Society of Anestesiologists (ASA) para ofrecer líneas generales uniformes. Se trata de considerar la gravedad de enfermedades sistémicas, disfunciones fisiológicas y anormalidades anatómicas. ${ }^{(6)}$

La anestesia, factor de gran influencia en todo el procedimiento anestésico quirúrgico, puede desencadenar alteraciones en el equilibrio hemodinámico. La palabra anestesia proviene de la palabra griega anaisthesis que literalmente quiere decir "sin sensación". (7)

La anestesia general es un estado inducido por um agente o una combinación de ellos que controlan la presión reversible de la función del sistema nervioso central (SNC), incluyendo la inconsciencia. Elementos básicos de la anestesia general incluyen disminución del nivel de conciencia o sueño, amnesia, analgesia, relajación muscular, respuesta motora disminuida a estímulos y reversibilidad. Obviamente, es importante mantener las funciones vitales (por ejemplo la cardiovascular, la respiratoria y la renal) durante toda la anestesia general. Los principales objetivos del acto anestésico son suplir la necesidad dolorosa durante la cirurgia manteniendo o no la conciencia; relajación muscular y proporcionar condiciones ideales para que el equipo quirúrgico pueda trabajar. ${ }^{(1)}$

En general, las condiciones clínicas preoperatorias, la extensión y tipo de cirugía, la técnica y la duración de la anestesia, las interocurrencias quirúrgicas y/o anestésicas y la eficacia de las medidas terapéuticas adoptadas están relacionadas con la frecuencia de complicaciones en el período postoperatorio inmediato. ${ }^{(8)}$

\section{OBJETIVO}

Identificar los diagnósticos de enfermería que se basan en la Taxonomía Il de la NANDA, en la SRPA, de pacientes adultos sometidos a anestesia general. 


\section{MATERIAL Y MÉTODO}

Se trata de un estudio descriptivo-exploratorio que sigue el método cuantitativo y delineamiento no experimental. Se llevó a cabo en una institución hospitalaria de tamaño especial (hasta quinientas camas) de la red que atiende a ambos sistemas, el público y privado, de la ciudad de Santos. El campo de investigación fue la sala de recuperación postanestésica, con ocho camas. La muestra incluyó treinta pacientes en recuperación anestésica. Los criterios de la muestra exigieron que el paciente fuese adulto clasificación ASA I sometido a cirugía electiva con anestesia general.

En primer lugar, se envió el proyecto de investigación a la institución con un pedido de autorización para la recogida de datos. Después del parecer de la Comisión de Ética e Investigación (CEP no 022) se comenzaron a recoger los datos. De la muestra participaron pacientes que, antes de la medicación preanestésica en el lugar del ingreso hospitalario, o sus acompañantes, después de haber aceptado el trabajo de investigación, estuvieron de acuerdo en participar. También se les pidió que firmasen el "término de consentimiento libre y esclarescido" conforme a las normas brasileñas establecidas por el Consejo Nacional de Salud, de acuerdo con la Resolución 196/96, que involucra investigación con seres humanos. Los datos se recogieron por medio de un instrumento estructurado (Apéndice A) y del prontuario médico del paciente.

El instrumento fue dividido en dos partes: parte I, referente a la identificación de la muestra, con datos tales como sexo, edad, diagnóstico médico, procedimiento anestésico-quirúrgico, tiempo de cirugía y de anestesia, recogidos del prontuario del paciente. La parte II, con información sobre las alteraciones durante la recuperación anestésica, recoge datos tales como las señales vitales, índice de Aldrete Kroulik, dolor, náuseas y vómitos. La señales vitales permiten medir alteraciones en la temperatura corporal, taquicardia, bradicardia, aumento o disminución de la tensión arterial (TA), frecuencia y ritmo respiratorio. El índice de Aldrete Kroulik, específico para medir las alteraciones durante la recuperación anestésica, evalúa cinco señales fisiológicas: actividad muscular, respiración, circulación, conciencia y saturación periférica de oxígeno $\left(\mathrm{SpO}_{2}\right)$. La actividad muscular considera la habilidad de mover los miembros espontáneamente o bajo mando, permite evaluar pacientes con bloqueos subaracnóideos o epidurales y evalúa los resíduos de relajantes musculares; la respiración verifica la capacidad de inspirar profundamente y toser, si la disnea es aparente; la circulación, de difícil valoración, verifica las diferencias de la TA de base del paciente en relación a los niveles presóricos preanestésicos; la conciencia valora el estado de alerta espontáneo - o cuando sea preciso - y la $\mathrm{SpO}_{2}$ permite evaluar el porcentaje de oxigenación periférica. $^{(7)}$

Los datos fueron evaluados cuando el paciente llegó a la SRPA, considerado momento cero, después de treinta minutos y después de sesenta minutos.

Los datos se organizaron en una planilla identificando los problemas de enfermería y relevamiento de diagnósticos de enfermería, con las características definidoras e identificación de los factores relacionados, de acuerdo con la NANDA ${ }^{(3)}$ y presentados en tablas con las frecuencias absolutas y relativas.

\section{RESULTADOS Y DISCUSIÓN}

Los resultados de la identificación de la muestra indican que hubo equivalencia entre el sexo femenino y el masculino entre pacientes de cuarenta y seis, cincuenta y cincuenta y un años. El diagnóstico médico más frecuente fue de hernia de disco lumbar, en la especialidad neurocirugía y el procedimiento anestésico-quirúrgico más frecuente microcirugía para 
corrección de hernia de disco. La especialidad de mayor frecuencia fue cirugía del aparato digestivo, órganos anexos y pared abdominal, con procedimientos anestésico-quirúrgicos del sistema digestivo en varios órganos y sistemas. Con relación al tiempo empleado en los procedimientos anestésico-quirúrgicos, el mayor fue para los intervalos de una a dos horas, considerados como procedimientos anestésicos-quirúrgicos medianos. ${ }^{(1)}$

\begin{tabular}{|c|c|c|c|}
\hline Señales Vital & & $\mathbf{N}^{\circ}$ & $\%$ \\
\hline & Hipotérmica & 24 & 80,0 \\
\hline Temperatura & Normotémica & 6 & 20,0 \\
\hline & Hipertérmica & 0 & 0,0 \\
\hline & Total & 30 & 100,0 \\
\hline & Óptima & 6 & 20,0 \\
\hline Tensión & Normal & 7 & 23,3 \\
\hline Arterial & Limítrofe & 6 & 20,0 \\
\hline Ingreso & Hipertensión & 11 & 36,7 \\
\hline & Total & 30 & 100 \\
\hline & Óptima & 6 & 20,0 \\
\hline Tensión & Normal & 9 & 30,0 \\
\hline Arterial & Limítrofe & 4 & 13,3 \\
\hline SRPA & Hipertensión & 11 & 36,7 \\
\hline & Total & 30 & 100 \\
\hline & Bradipneica & 3 & 10,0 \\
\hline Respiración & Eupneica & 14 & 46,7 \\
\hline & Taquipneica & 13 & 43,3 \\
\hline & Total & 30 & 100,0 \\
\hline & Bradicárdico & 3 & 10,0 \\
\hline Pulso & Normocárdico & 24 & 80,0 \\
\hline & Taquicárdico & 3 & 10,0 \\
\hline & Total & 30 & 100,0 \\
\hline
\end{tabular}

La tabla 1 muestra la valoración de las señales vitales. Veinticuatro pacientes $(80,0 \%)$ estaban hipotérmicos con temperaturas entre 35 y $35,5^{\circ} \mathrm{C}$. La hipotermia está determinada por la temperatura corporal inferior a $36^{\circ} \mathrm{C}$; puede considerarse como leve (entre $32^{\circ} \mathrm{C}$ y $35^{\circ} \mathrm{C}$ ), media 0 moderada (entre $28^{\circ} \mathrm{C}$ y $32^{\circ} \mathrm{C}$ ) y grave o severa (inferior a $28^{\circ} \mathrm{C}$ ) ${ }^{(10)}$. La pérdida de calor es común en todos los pacientes durante la anestesia general porque los anestésicos alteran el centro de termorregulación del hipotálamo, inhiben los temblores y producen vasodilatación periférica. Durante la anestesia el paciente no muestra respuestas de regulación térmica porque el reflejo está inhibido. Durante la recuperación de la anestesia la inhibición desaparece y los temblores comienzan cuando la temperatura está por debajo del umbral de la regulación térmica. ${ }^{(9,10)}$

Mendoza, Peniche ${ }^{(11)}$ relatan que la caída de temperatura es inherente al proceso quirúrgico de los pacientes de la tercera edad debido a la alteración del sistema termorregulador por causa de drogas o de la anestesia. Asimismo, factores como la posición, temperatura de la sala de operaciones, calentamiento de los fluidos y uso de mantas también influyen en la temperatura corporal. Luego las intervenciones de enfermería deben ser preventivas durante el período anestésico-quirúrgico para no perjudicar el postoperatorio. 
A pesar de que la hipotermia sea una complicación común en el período de recuperación anestésica, Gotardo, Silveira, Galvão ${ }^{(12)}$ evidencian la escasez de trabajos sobre este asunto. Al realizar una búsqueda bibliográfica manual de revistas clasificadas como Qualis $B$ y $C$ internacionales y $A$ y $B$ nacionales, se hallaron doscientos noventa y siete ejemplares de los cuales cuatro trataban de la hipotermia como DE y principales factores relacionados y uno recomendaba prevenir tal complicación.

Con relación a la TA, once pacientes (36,7\%) presentaron hipertensión arterial en el ingreso hospitalario y once $(36,7 \%)$ en la SRPA, según la tabla 1 . La TA está considerada como óptima (< 120x80 mmHg), normal $(<130 \times 85 \mathrm{mmHg})$, limítrofe $(130-139$ / 85-89 mmHg), e hipertensión, fase I - ligera (140-159 / 90-99 mmHg), fase II - moderada (160-179 / 100-109 $\mathrm{mmHg})$ y fase III -grave $(\geq 180 / \geq 110 \mathrm{mmHg})$. ${ }^{(13)}$

Para Rocha, Rocha ${ }^{(14)}$, las variaciones en los niveles de tensión pueden ocurrir en secuencias distintas del acto quirúrgico, elevándose durante la inducción anestésica, disminuyendo con la profundización de la anestesia y aumentando nuevamente en el período de recuperación. Las oscilaciones ascendentes con media de $20 \mathrm{~mm} \mathrm{Hg}$ por más de quince minutos predisponen al paciente a las complicaciones tanto en el período intraoperatorio como en el postoperatorio.

Se observó taquipnea en trece $(43,3 \%)$ pacientes de la muestra. Esta manifestación pudo explicarse por la acumulación de dióxido de carbono (PCO2) debido a los temblores y, a veces, por la excitación al despertarse de la anestesia, dolor y ansiedad, lo cual conlleva a la taquipnea como una manifestación secundaria de compensación para eliminar el dióxido de carbono. ${ }^{(7)}$

Tabla 2: Distribución de la frecuencia de pacientes en la SRPA, con anestesia general, según el índice de Aldrete Kroulik, Santos, 2007.

\begin{tabular}{|c|c|c|c|c|c|c|}
\hline \multirow{2}{*}{$\begin{array}{l}\text { Actividad } \\
\text { Muscular }\end{array}$} & \multicolumn{2}{|c|}{$0 \mathrm{~min}$} & \multicolumn{2}{|c|}{30 mim } & \multicolumn{2}{|c|}{60 min* } \\
\hline & $\mathbf{N}^{\circ}$ & $\%$ & $\mathbf{N}^{\circ}$ & $\%$ & $\mathbf{N}^{\circ}$ & $\%$ \\
\hline 2 & 10 & 33,3 & 23 & 76,7 & 19 & 86,4 \\
\hline 1 & 17 & 56,7 & 6 & 20,0 & 3 & 13,6 \\
\hline 0 & 3 & 10,0 & 1 & 3,3 & 0 & 0,0 \\
\hline Total & 30 & 100,0 & 30 & 100,0 & 22 & 100,0 \\
\hline \multicolumn{7}{|c|}{ Respiración } \\
\hline 2 & 22 & 73,4 & 30 & 100,0 & 22 & 100,0 \\
\hline 1 & 7 & 23,3 & 0 & 0,0 & 0 & 0,0 \\
\hline 0 & 1 & 3,3 & 0 & 0,0 & 0 & 0,0 \\
\hline Total & 30 & 100,0 & 30 & 100,0 & 22 & 100,0 \\
\hline \multicolumn{7}{|c|}{ Circulación } \\
\hline 2 & 28 & 93,3 & 29 & $\overline{96,7}$ & 22 & 100,0 \\
\hline 1 & 2 & 6,7 & 1 & 3,3 & 0 & 0,0 \\
\hline 0 & 0 & 0,0 & 0 & 0,0 & 0 & 0,0 \\
\hline Total & 30 & 100,0 & 30 & 100,0 & 22 & 100,0 \\
\hline \multicolumn{7}{|c|}{ Conciencia } \\
\hline 2 & 7 & 22,3 & 23 & 76,7 & 20 & 90,9 \\
\hline 1 & 19 & 63,4 & 6 & 20,0 & 2 & 9,1 \\
\hline 0 & 4 & 13,3 & 1 & 3,3 & $\overline{0}$ & 0,0 \\
\hline Total & 30 & 100,0 & 30 & 100,0 & 22 & 100,0 \\
\hline SpO2 & & & & & & \\
\hline
\end{tabular}




\begin{tabular}{|l|l|l|l|l|l|l|}
\hline $\mathbf{2}$ & 17 & 56,7 & 24 & 80,0 & 19 & 86,6 \\
\hline $\mathbf{1}$ & 13 & 43,3 & 6 & 20,0 & 3 & 13,4 \\
\hline $\mathbf{0}$ & 0 & 0,0 & 0 & 0,0 & 0 & 0,0 \\
\hline Total & 30 & 100,0 & 30 & 100,0 & 22 & 100,0 \\
\hline Resultado & & & & & & \\
\hline $\mathbf{0}-\mathbf{5}$ & 6 & 20,0 & 0 & 0,0 & 0 & 0,0 \\
\hline $\mathbf{6}-\mathbf{8}$ & 16 & 53,3 & 7 & 23,3 & 1 & 4,5 \\
\hline $\mathbf{9}-\mathbf{1 0}$ & 8 & 26,7 & 23 & 76,7 & 21 & 95,5 \\
\hline Total & 30 & 100,0 & 30 & 100,0 & 22 & 100,0 \\
\hline
\end{tabular}

Para el Índice de Aldrete Kroulik, en la Tabla 2, se observa que al llegar a la SRPA, en el minuto cero, dieciséis pacientes $(53,3 \%)$ tuvieron entre seis y ocho puntos, es decir, presentaron de dos a cuatro alteraciones en alguna señal fisiológica evaluada por el índice, ya sea de actividad muscular, conciencia, respiración, circulación y/ o $\mathrm{SpO}_{2}$. Estos valores indican que se debe de vigilar al paciente y no permitir su alta de la SRPA. ${ }^{(5,7)}$ La señal fisiológica de conciencia presentó la mayor frecuencia de nota uno, con diecinueve $(63,4 \%)$, seguida de actividad muscular y $\mathrm{SpO}_{2}$, con diecisiete $(56,7 \%)$ y trece $(43,3 \%)$ respectivamente. Narcóticos, sedativos o anestésicos residuales pueden causar alteraciones en el estado mental y el relajante muscular residual conduce a la debilidad muscular durante el período de recuperación anestésica. ${ }^{(5,7-8)}$

En treinta minutos veintitrés pacientes $(76,7 \%)$ obtuvieron entre nueve y diez puntos, lo cual significa sólo una alteración moderada, con nota uno. Se observó actividad muscular o conciencia $\circ \mathrm{SpO}_{2}$ alteradas en seis $(20,0 \%)$ de los pacientes.

En sesenta minutos, veintiún pacientes $(95,5 \%)$ también presentaron entre nueve y diez puntos. Solamente veintidós pacientes permanecieron sesenta minutos en la SRPA, por tener alta antes de los sesenta ya que tenían entre nueve y diez puntos del índice de Aldrete Kroulik.

Con respecto a la valoración del dolor, diecisiete $(56,7 \%)$ pacientes llegaron a la SRPA con dolores y la minoría continuó con dolores durante treinta y sesenta minutos consecutivos. En un trabajo de revisión de la literatura de artículos indexados sobre las complicaciones del paciente quirúrgico de edad, Mendoza, Peniche ${ }^{(11)}$ demostró que $50 \%$ de los estudios relatan el dolor como una complicación en el período de recuperación anestésica, seguido de náuseas y vómitos en $14,3 \%$ de los estudios.

Meireles et al ${ }^{(15)}$, en un estudio realizado en la unidad de cuidados intensivos en pacientes en el postoperatorio, demostró que $43 \%$ continuaban con dolores álgidos aún después de haber recibido analgésicos hasta seis horas antes; consideraban dolor moderado en $23 \%$ e intenso en $42 \%$ de los casos.

En cuanto a las náuseas, tres pacientes (10,0\%) llegaron a la SRPA con náuseas. De estos três, sólo uno tuvo vómitos a los sesenta minutos de permanencia. Las náuseas y los vómitos son complicaciones frecuentes en el período de recuperación anestésica y se desencadenan tanto por dolor como por medicaciones opiáceas, pudiendo evitarse con medicaciones antieméticas durante el período intraoperatorio. ${ }^{(7)}$ 
Tabla 3: Distribución de la frecuencia de los pacientes en SRPA con anestesia general según el diagnóstico de enfermería, Santos, 2007.

\begin{tabular}{|c|c|c|c|c|c|}
\hline $\begin{array}{l}\text { Problema } \\
\text { Identificado }\end{array}$ & $\begin{array}{l}\text { Diagnóstico } \\
\text { de Enfermería }\end{array}$ & $\begin{array}{l}\text { Características } \\
\text { Definidoras }\end{array}$ & $\begin{array}{l}\text { Factores } \\
\text { Relacionados }\end{array}$ & $\mathbf{N}$ & $\%$ \\
\hline $\begin{array}{l}\text { Temperatura } \\
\text { inferior a } 36^{\circ} \mathrm{C}\end{array}$ & Hipotermia & $\begin{array}{ll}\text { Temblor, piel } \\
\text { fría, reducción } \\
\text { de } \quad \text { la } \\
\text { temperatura } \\
\text { corporal. }\end{array}$ & $\begin{array}{l}\text { Exposición al } \\
\text { ambiente } \\
\text { fresco, ropa } \\
\text { inadecuada. }\end{array}$ & 24 & 80,0 \\
\hline Náuseas & Náuseas & $\begin{array}{l}\text { Relato de } \\
\text { náuseas, } \\
\text { salivación } \\
\text { aumentada. }\end{array}$ & $\begin{array}{l}\text { Factores } \\
\text { psicológicos, } \\
\text { irritación } \\
\text { gástrica por } \\
\text { medicamentos. }\end{array}$ & 3 & 10,0 \\
\hline Dolor & Dolor agudo & $\begin{array}{l}\text { Relato verbal, } \\
\text { expresión } \\
\text { facial. }\end{array}$ & $\begin{array}{l}\text { Agentes } \\
\text { lesivos } \\
\text { (físicos, } \\
\text { biológicos). }\end{array}$ & 20 & 67,9 \\
\hline
\end{tabular}

Después de haber identificado los problemas, se relevaron los diagnósticos de enfermería de mayor frecuencia, según la NANDA ${ }^{(3)}$, con las características definidoras y los factores relacionados, siendo hipotermia con veinticuatro $(80,0 \%)$ de los pacientes, seguido de dolor agudo en veinte $(67,9 \%)$ y náuseas en tres $(10,0 \%)$, en conformidad con la Tabla 3.

Rossi et al ${ }^{(16)}$ elaboraron un estudio con miras a identificar los DE más frecuentes en el post-operatorio inmediato, e identificaron disciséis DE, entre ellos hipotermia en $67,8 \%$ y dolor en $32,2 \%$ de los casos. Galdeano et al ${ }^{(17)}$ también elaboraron un estudio con el objetivo de identificar los DE en pacientes adultos, en el período transoperatorio de cirugía cardíaca, e identificaron once DE: $94,1 \%$ de los casos presentaron riesgo por temperatura corporal alterada.

Biazzotto et al ${ }^{(18)}$ señalan las siguientes complicaciones de la hipotermia: aumento de la mortalidad cardíaca, coagulopatía, aumento de la incidencia de infección en el sitio quirúrgico, alteraciones hormonales, aumento de los efectos de las drogas anestésicas, aumento de la permanencia del paciente en la SRPA y presencia de temblores musculares.

La American Society of PeriAnesthesia Nurses ${ }^{(19)}$ cuenta con directrices para prevenir y tratar la hipotermia durante el perioperatorio. Deben evaluarse factores de riesgo tales como extremos de edad, tipo de procedimiento y enfermedades asociadas, verificarse las señales de hipotermia y, aún en pacientes con normotermia, deben tomarse medidas preventivas como usar sábanas de algodón, calcetines y gorro, exposición limitada y aumentar la temperatura ambiente. La mensuración y cuidados deben aplicarse al entrar en el ambiente quirúrgico, en la sala de operaciones y en la sala de recuperación postanestésica cada treinta minutos, hasta la normotermia.

El dolor en el período postoperatorio inmediato, considerado como dolor agudo, presenta respuestas fisiológicas, incluyendo disfunciones pulmonares, cardiovasculares, urinarias, metabólicas y neuroendocrinas, que causan estimulación simpática con consiguiente taquicardia, aumento del volumen sistólico, trabajo cardíaco y consumo de oxígeno por el miocardio y trombosis de venas profundas debido a la disminución de los movimientos con miedo de sentir dolor. Manifestaciones de exacerbación del sistema simpático acarrean vasoconstricción y consiguiente hipertensión arterial, manifestándose con una disminución 
de perfusión periférica, oxigenación pulmonar, disminución del débito urinario y agitación, todas ocasionadas por la mala perfusión tecidual. ${ }^{(20)}$

El alivio del dolor en el período postoperatorio inmediato (PO) promueve mejor rehabilitación como disminución de las complicaciones pulmonares, preservación de la función miocárdica, deambulación precoz, menor incidencia de tromboembolismo, reducción del tiempo de ingreso hospitalario, además de comodidad para el paciente. ${ }^{(21)}$

De tal forma, se observa que tanto la hipotermia como el dolor son complicaciones comunes en el período de recuperación anestésica, y éstas, a su vez, deben ser prevenidas, pues acarrean complicaciones sistémicas importantes.

\section{CONCLUSIÓN}

Los resultados de este estudio han permitido concluir que de las alteraciones evidenciadas, los diagnósticos de enfermería de mayor frecuencia son hipotermia y dolor agudo. A pesar de tratarse de un estudio preliminar, con pequeño recorte de una realidad infinita, los resultados y los estudios realizados colocan en evidencia que la hipotermia y el dolor agudo son comunes en los procedimientos anestésico-quirúrgicos entre las complicaciones posibles durante el período de recuperación anestésica.

Estas alteraciones pueden prevenirse planificando la asistencia de enfermería, tanto de calentamiento en el período anestésico-quirúrgico como de control y valoración del dolor en el período de recuperación anestésica.

El rol fundamental de los enfermeros es el de prevenir las complicaciones y esta prevención debe ser planificada en el período preoperatorio, puesta en práctica en el transoperatorio y evaluada durante todo perioperatorio.

La identificación de los problemas y la determinación de los DE permiten promover la asistencia de enfermería individualizada, planificada, y ayuda a prevenir daños en el postoperatorio.

\section{REFERENCIAS BIBLIOGRÁFICAS}

1. Possari JF. Centro Cirúrgico: Planejamento, Organização e Gestão. São Paulo: látria; 2004.

2. Horta WA. Processo de enfermagem. 16ª ed. São Paulo: EPU; 2005.

3. North American Nursing Diagnosis Association. NANDA. Diagnósticos de Enfermagem da NANDA: definições e classificação. Porto Alegre: Artmed; 2005-2006.

4. Prado KG, Silva LF, Graciano LP, Domingues LG, Telles Filho PC, Michigami RCM et AL. Centro de recuperação pós-anestésico: observação, análise e comparação. Rev Latino-Am de Enferm 1998; 6 (3): 123-5. [Acesso em 08 de mar. 2007]

Disponível em: http//: www.scielo.br/pdf/rLae/v6n3/13899.pdf.

5. Possari JF. Assistência de Enfermagem na Recuperação Pós-Anestésica (RPA). 2ª ed. São Paulo: látria; 2006.

6. Sociedade Brasileira de Enfermeiros de Centro Cirúrgico, Recuperação Anestésica e Centro de Material e Esterilização. SOBECC. Práticas Recomendadas da SOBECC. 4a ed. São Paulo, 2007.

7. Meeker $\mathrm{MH}$, Rothrock JC. Alexander. Cuidados de Enfermagem ao Paciente Cirúrgico. $10^{a}$ ed. Rio de Janeiro: Guanabara Koogan; 1997. Cap. 6 e 8. 
8. Mauro G, Cardoso AR. In: Congiani LM, Posso PI, Poterio GMB, Nogueira CS. Tratado de anestesiologia. Serviço de Anestesia do Estado de São Paulo. 6a ed. São Paulo: Atheneu; 2006. vol. 2, cap. 105, p. 1351-9.

9. Albergaria VF et al. Tremores intra e pós-operatório: prevenção e tratamento farmacológico. Rev Bras de Anestesiologia 2007; 57(4). [Acesso em 01 de mar 2007] Disponível em: http//: www.scielo.br/pdf/v57n4.

10. Duke J. Segredos em anestesiologia. 2ª edição. Porto Alegre: Artmed; 2003.

11. Mendoza IYQ, Peniche ACG. Complicações do paciente cirúrgico idoso no período de recuperação anestésica: revisão da literatura. Rev Soc Bras de Enfermeiros de Centro Cirúrgico, Recuperação Anestésica e Centro de Material e Esterilização 2008; 13 (1):25-31.

12. Gotardo JM, Silveira RCCP, Galvão CM. Hipotermia no perioperatório: análise de produção científica nacional de enfermagem. Rev Soc Bras Enfermeiros de Centro Cirúrgico, Recuperação Anestésica e Centro de Material e Esterilização 2008; 13 (2): 40-8.

13. Sociedade Brasileira de Cardiologia. Sociedade Brasileira de Hipertensão. Sociedade Brasileira de Nefrologia. IV Diretrizes Brasileiras de Hipertensão Arterial. Arq Bras Cardiol 2004; (supl.4): 1-40. [Citado em 30 de jul. 2004] Disponível em: http//:www.scielo.br.

14. Rocha JC, Rocha AT. Abordagem pré-operatória do paciente hipertenso: riscos e orientações. Rev Soc Cardiol. 2000; 10 (3):311-6.

15. Meireles CCS, Gomes SFS, Bezerra SL, Soares CS, Mota DDCF, Pimenta CAM. Dor em UTI: prevalência e características da queixa dos pacientes. Rev Soc Bras Enfermeiros de Centro Cirúrgico, Recuperação Anestésica e Centro de Material e Esterilização 2006; 11 (4):37-41.

16. Rossi LA, Torrati FG, Carvalho EC, Manfrim A, Silva DF. Diagnósticos de enfermagem do paciente no período pós-operatório imediato. Rev Esc Enferm USP, 2000, 34 (2): 154-64.

17. Galdeano LE, Rossi LA, Nobre LF, Ignacio DS. Diagnósticos de enfermagem de pacientes no período transoperatório de cirurgia cardíaca. Rev Latino-Am Enferm USP, 2003, 11 (2): 99-206.

18. Biazzotto CB, Brudniewski M, Schmidt AP, Auler Junior JOC. Hipotermia no período perioperatório. Rev Bras Anestesiol 2006; 56 (1): 89-106.

19. American Society of PeriAnesthesia Nurses. Clinical guideline for the prevention of unplanned perioperative hypothermia. J Perianesth Nurs. 2001; 16 (5): 305-14.

20. Ready LB, Edwards WT. Tratamento de dor aguda. Rio de janeiro: Revinter; 1995.

21. Sakata KR, Issy MA. Guias de medicina ambulatorial e hospitalar. São Paulo: Manole; 2004. 


\section{APÉNDICE A}

INSTRUMENTO DE RECOGIDA DE DATOS

\begin{tabular}{|c|c|c|c|c|c|c|c|c|c|}
\hline PACIENTES & \multicolumn{3}{|l|}{1} & \multicolumn{3}{|l|}{2} & \multicolumn{3}{|l|}{3} \\
\hline \multicolumn{10}{|l|}{$\begin{array}{l}\text { Parte I: identificación de la } \\
\text { muestra }\end{array}$} \\
\hline \multicolumn{10}{|l|}{ Sexo } \\
\hline \multicolumn{10}{|l|}{ Edad } \\
\hline \multicolumn{10}{|l|}{ Diagnóstico médico } \\
\hline \multicolumn{10}{|l|}{$\begin{array}{l}\text { Intervención } \quad \text { anestésico- } \\
\text { quirúrgica }\end{array}$} \\
\hline \multicolumn{10}{|l|}{ Tiempo de cirugía } \\
\hline \multicolumn{10}{|l|}{ Tiempo de anestesia } \\
\hline \multicolumn{10}{|l|}{ Parte II: complicaciones } \\
\hline \multicolumn{10}{|l|}{ Señales vitales } \\
\hline \multicolumn{10}{|l|}{$\mathrm{T}$} \\
\hline \multicolumn{10}{|l|}{$\mathrm{P}$} \\
\hline \multicolumn{10}{|l|}{$\mathrm{R}$} \\
\hline \multicolumn{10}{|l|}{ TA ingreso hospitalario } \\
\hline \multicolumn{10}{|l|}{ TA a la llegada a la SRPA } \\
\hline Índice de Aldrete Kroulik & 0 & 30 & 60 & 0 & 30 & 60 & 0 & 30 & 60 \\
\hline \multicolumn{10}{|l|}{ Actividad muscular } \\
\hline \multicolumn{10}{|l|}{ Respiración } \\
\hline \multicolumn{10}{|l|}{ Circulación } \\
\hline \multicolumn{10}{|l|}{ Conciencia } \\
\hline \multicolumn{10}{|l|}{$\mathrm{SpO}_{2}$} \\
\hline \multicolumn{10}{|l|}{ Total } \\
\hline \multicolumn{10}{|l|}{ Náuseas } \\
\hline \multicolumn{10}{|l|}{ Vómitos } \\
\hline Dolor & & & & & & & & & \\
\hline
\end{tabular}

ISSN 1695-6141 